\title{
Comparative Evaluation of Dexmedetomidine Versus Magnesium Sulphate on The Adequacy of Hypotensive Anesthesia and Post-Operative Recovery for Patients Undergoing Endoscopic Transnasal Transsphenoidal Pituitary Tumor Resection
}

\author{
Ahmed Mohammed Sonbol*, Sameh Ghareeb Ahmed \\ Department of Anesthesiology, Faculty of medicine, Mansoura University, Egypt. \\ *Corresponding author: Ahmed Mohammed Sonbol, Mobile: (+20) 01003735129. E-Mail: dr.sonbol@ gmail.com
}

\begin{abstract}
Background: Perioperative use of magnesium sulfate $\left(\mathrm{MgSO}_{4}\right)$, dexmedetomidine, have been tried in order to provide beneficial clinical effects during general anesthesia (GA). However, few literature discussed it with varying results. Several clinical researches have showed that usage of $\mathrm{MgSO}_{4}$ infusion was associated with a reduction in anesthetic requirement and postsurgical analgesic consumption during GA.

Objective: This study aimed to assess the pharmacologic effects of the use of dexmedetomidine and $\mathrm{MgSO}_{4}$ on anesthetic requirement, intra operative haemodynamics stability and postsurgical analgesic effects on the adequacy of hypotensive anesthesia during transsphenoidal resection of pituitary tumours.

Patients and methods: A total of 110 cases were enrolled in this prospective study. They were randomized into 2 groups: Group D (55 cases) that was commenced on dexmedetomidine, and group M (55 cases) which received $\mathrm{MgSO}_{4}$ Results: The mean values of Boezaart score were significantly decreased in Group D in comparison to group M. In addition, isoflurane and propranolol consumption showed a significant decrease in group D. However, blood loss showed no significant difference when comparing the same groups. Group D expressed significantly longer emergence and extubation times compared to Group $\mathrm{M}$.
\end{abstract}

Conclusion: Dexmedetomidine appears to be superior compared to magnesium sulphate in achieving hypotensive anesthesia during pituitary surgery.

Key words: Dexmedetomidine; Magnesium sulphate; Hypotensive anesthesia; Pituitary surgery.

\section{INTRODUCTION}

Neuroanesthesia has some basic principles including smooth induction, hemodynamic stability, maintaining cerebral perfusion, and providing optimal operative conditions to facilitate good exposure for the surgeon. Smooth emergence is of great importance such as smooth induction as it allows early evaluation of the neurological functions after surgery ${ }^{(1)}$.

Trans-sphenoidal excision of pituitary tumours is a common neurosurgical approach, as it accounts for $20 \%$ of all intracranial operations in most neurosurgical centers (2). Anesthetic management for such cases represents a challenge to anesthesiologists as nasal speculum insertion during the procedure results in a strong nociceptive stimulation, which in turn will lead to tachycardia and hypertension. This will lead to bleeding and difficult visualization of the operative field ${ }^{(3,4)}$.

Multiple drugs are recommended to obtain controlled hypotension during neurosurgical procedures including; beta-blockers, sodium nitroprusside, nitroglycerine, increasing the dosages of inhaled anesthetic agents, alpha- 2 agonists and $\mathrm{MgSO}_{4}{ }^{(5)}$.

Dexmedetomidine is an alpha-II adrenergic agonist, which has sedative and analgesic, as well as anesthetic sparing effect, without any negative impact on the respiratory center. It modulates pin signals transmission via acting on both spinal and supraspinal regions ${ }^{(1)}$. Dexmedetomidine is associates with decrease of inhaled anesthetic requirements. Additionally, in neurosurgical patients, it helps to stabilize intracranial pressure along with intraoperative hemodynamic stability especially on intubation and extubation. It also to decreases anesthetic and opioid consumption ${ }^{(6)}$.

Magnesium sulfate $\left(\mathrm{MgSO}_{4}\right)$ is a NMDA receptor antagonist with antinociceptive effects ${ }^{(7)}$. It has the ability to induce deliberate hypotension by blocking of trans-membrane calcium ATPase and $\mathrm{Na}^{+} / \mathrm{K}^{+}$-ATPase ion channels. In addition, $\mathrm{Mg}^{++}$suppresses the discharge of norepinephrine. It also has a direct vasodilator effect by increasing prostacyclin synthesis, as well as inhibiting ACE activity. A lot of investigations have displayed that $\mathrm{MgSO}_{4}$ infusion throughout general anesthesia was accompanied by a reduction in anesthetic need and postsurgical analgesic requirement ${ }^{(8)}$.

The current study aimed to compare between dexmedetomidine and $\mathrm{MgSO}_{4}$ in the context of adequacy of hypotensive anesthesia during trans-sphenoidal excision of pituitary tumours.

\section{PATIENTS AND METHODS}

This was a prospective randomized study carried out at Mansoura University Hospitals within a period of two years, starting from December 2018 till December 2020.

Inclusion criteria: Age from 18 and 65 years who were electively prepared for pituitary tumour resection.

Exclusion criteria: patients with Glasgow Coma Scale (GCS) < 15, American Society of Anesthesiologists (ASA) score > II, preoperative hear rate $<50 \mathrm{bpm}$, firstor second-degree heart block, allergy to the study 
medications, pregnancy and patients commenced on beta blockers, alpha-methyl dopa, clonidine, or other alpha-2 agonists.

Sample size was measured according to a prior research (9), where at the time of nasal speculum insertion, the rising in MAP was $9.4 \%$ in dexmedetomidine group, on G power to determine a change of twenty percent in MAP among the magnesium sulphate and the Dexmedetomidine groups. The needed number for $\alpha$ error of 0.05 and power of eighty percent was measured to be 55 cases in each group.

\section{This study was carried out on two groups:}

Group D: included 55 cases who were commenced on dexmedetomidine $(1 \mathrm{mcg} / \mathrm{kg}$ over ten $\min$ then, a maintenance of $0.5 \mathrm{mcg} / \mathrm{kg} / \mathrm{hr}$ ), and

Group M: included the remaining 55 cases who received $\mathrm{MGSO}_{4}$. In (M group), cases received $40 \mathrm{mg} / \mathrm{kg} \mathrm{MgSO}$ in $100 \mathrm{ml}$ saline solution over ten min as the IV loading dosage 10 min prior to induction, with a consequent 10 $15 \mathrm{mg} / \mathrm{kg} / \mathrm{h}$ infusion throughout the operation.

Both drugs were prepared by a staff nurse and an anesthesiologist who were blind to both drug and patient allocation. Both of these regimens were started ten min before anesthetic induction.

At neurosurgical operative theater, the standard patient monitoring was ensured including noninvasive arterial pressure, pulse and oxygen saturation. Additionally, an intravenous line was established. Before induction, midazolam $(0.03 \mathrm{mg} / \mathrm{kg})$, fentanyl (1 $\mathrm{mic} / \mathrm{kg}$ ) were given to all cases. Induction of anesthesia was done by propofol $(0.5-2 \mathrm{mg} / \mathrm{kg})$, while atracurium $(0.5 \mathrm{mg} / \mathrm{kg})$ was used for neuromuscular blockade. Then, direct laryngoscopy with insertion of the proper size endotracheal tube was done.

Anesthesia was kept with air in oxygen (50\%:50\%) and isoflurane. IPPV with a TV of 7 to 8 $\mathrm{ml} / \mathrm{kg} \mathrm{BW}$ was performed to keep end tidal $\mathrm{CO}_{2}$ between 30 and $35 \mathrm{~mm} \mathrm{Hg}$. Also, a moist cotton gauze was used to pack the posterior pharynx under direct laryngoscopy. If any rise in the HR or MBP > 20\%, compared to the baseline, the initial isoflurane $0.5 \%$ end-tidal level was raised by $0.2 \%$ every four minutes up to a maximum of $2 \%$ end-tidal level. If no response was detected, either nitroglycerine (increment $0.1-0.25 \mathrm{mg}$ ) or propranolol (increment 0.5 up to $2 \mathrm{mg}$ ) was administered.

Both blood pressure and pulse were measured and monitored. They were recorded at the next time points; baseline, before intubation, following intubation, insertion of nasal speculum, at 15 minutes, 30 minutes, then after 30 minutes till end of surgery, pre-extubation, and post-extubation. If hypotension was detected (defined as systolic blood pressure $<90 \mathrm{mmHg}$ ), ephedrine $5 \mathrm{mg}$ was given by IV route. Besides, bradycardia, described as HR below $50 \mathrm{bpm}$, was managed by intravenous atropine $(0.02 \mathrm{mg} / \mathrm{kg})$.

Boezaart bleeding scale was utilized to assess the quality of surgical field regarding bleeding as follows; (0) virtually bloodless field, (1) slight oozing for which suctioning isn't essential, (2) minimal bleeding requiring occasional suctioning with no interference with the surgical field, (3) moderate bleeding requiring usual suctioning that improves the visual field, (4) heavy bleeding with regular suctioning, and the surgical field worsens following suction was withdrawn, (5) extensive uncontrollable blood loss faster than suctioning ${ }^{(10)}$.

Blood loss, fluid intake, and UOP were measure and recorded. Both $\mathrm{MgSO}_{4}$ and dexmedetomidine were stopped ten min prior to the termination of operation, which was described as the time point once the neurosurgeon removed the nasal speculum. After that, isoflurane was ceased, and neuromuscular blockade was backed by utilizing both neostigmine and atropine. The total doses of isoflurane, propranolol, and nitroglycerine were calculated and recorded. Total isoflurane dose was calculated as recommended by Biro ${ }^{(11)}$. Additionally, both emergence and extubation times were recorded. All patients were then moved to the PACU, where observation was continued by an examiner and PACU nurse, neither of whom was aware of the anesthetic regimen. Post-operative pain was evaluated by VAS, with 0 for no pain, and 10 for the worst pain ever ${ }^{(12)}$ at 15,30 , and $60 \mathrm{~min}$ following tracheal extubation. If VAS was > 40, ketorolac $30 \mathrm{mg}$ IV was given.

Entire cases were discharged when reaching a score of nine by utilizing a modified Aldrete scoring system as shown in table (1).

Table (1) Modified Aldrete Score (MAS) ${ }^{(13)}$

\begin{tabular}{|l|l|c|}
\hline Criteria & \multicolumn{1}{|c|}{ Characteristics } & Points \\
\hline Activity & Able to move 4 extremities & 2 \\
\cline { 2 - 3 } & Able to move 2 extremities & 1 \\
\cline { 2 - 3 } & Unable to move extremities & 0 \\
\hline \multirow{4}{*}{ Circulation } & Able to breathe deeply and & 2 \\
\cline { 2 - 3 } & Dyspnea or limited breathing & 1 \\
\cline { 2 - 3 } & Apneic & 0 \\
\cline { 2 - 3 } & BP $+/-20 \%$ of pre-anesthetic & 2 \\
\cline { 2 - 3 } & BP $+/-20-49 \%$ of pre- & 1 \\
\hline \multirow{4}{*}{ Consciousness $+/-50 \%$ of pre-anesthetic } & 0 \\
\hline \multirow{3}{*}{$\begin{array}{l}\text { Oxygen } \\
\text { saturation }\end{array}$} & Fully awake & 2 \\
\cline { 2 - 3 } & Arousable on calling & 1 \\
\cline { 2 - 3 } & Not responding & 0 \\
\cline { 2 - 3 } & Able to maintain O2 & 2 \\
\cline { 2 - 3 } & Needs oxygen to maintain & 1 \\
\cline { 2 - 3 } & O2 saturation $<90 \%$ even & 0 \\
\hline
\end{tabular}

Comparing intraoperative hemodynamic parameters along with Boezaart bleeding scale were our primary outcomes. Secondary outcomes included inhalational anesthetic requirement, hypotensive agent doses required, emergence time, extubation time, postoperative pain, and analgesic requirements.

\section{Ethical consent:}

The approval of the study was got from IRB of Faculty of Medicine, Mansoura University prior beginning the research and an informed written 
consent was taken from each participant in the study. Number of ethical approval is R.20.08.966. This work has been carried out in accordance with The Code of Ethics of the World Medical Association (Declaration of Helsinki) for studies involving humans.

The researcher elucidated the objective and aim of the study to the subjects involved within study. The researcher guaranteed keeping anonymity and confidentiality of the subject's data. Subjects were told that they were allowable to choose to share or not in the research and that they had the right to leave the research at any time without explaining causes.

\section{Statistical analysis}

IBM's SPSS statistics for windows (version 20) was utilized for assessment of the gathered information. Shapiro-Wilk test was used to check the normality of the data distribution. Quantitative variables were expressed as mean $\pm \mathrm{SD}$, whereas categorical variables were expressed as frequency and percent. Independent sample T and Mann Whitney tests were utilized for comparison of parametric and non-parametric continuous data correspondingly. In terms of pair-wise comparison of data, the follow up values were compared to the matching basal level by utilizing paired samples $\mathrm{T}$ test or Wilcoxon matched pairs signed ranks test.
Fisher exact and Chi square tests were utilized for inter-group comparison of nominal data by utilizing the crosstabs functions. $\mathrm{P} \leq 0.05$ is considered significant.

\section{RESULTS}

This was a prospective randomized study carried out at Mansoura University hospitals during a period of two years, starting from December 2018 till December 2020. This study was carried out on two groups: Group D: included 55 cases who were commenced on dexmedetomidine and Group M: included the remaining 55 cases who received $\mathrm{MGSO}_{4}$. Starting with demographics, age and sex weren't significantly different among both groups ( $\mathrm{p}=0.640$ and 0.565 correspondingly). The mean age of the comprised cases was 46.62 and 45.75 years in groups $\mathrm{D}$ and $\mathrm{M}$ correspondingly. Males represented 58.2 and $52.7 \%$ of the included population in both groups correspondingly. Additionally, the mean values of BMI were 30.1 and $29.77 \mathrm{~kg} / \mathrm{m}^{2}$ in the study groups correspondingly $(\mathrm{p}=$ 0.693). Diabetes mellitus was present in 3.6 and 9.1 of cases, whereas hypertension was prevalent in 20 and $18.2 \%$ of cases in the same groups correspondingly. Operative time didn't significantly differ between the studied groups (160.91 and 153 minutes correspondingly $-\mathrm{p}=0.155)$ as demonstrated in table (2).

Table (2): Sociodemographic features, medical history and operative duration of the study groups

\begin{tabular}{|c|c|c|c|c|c|}
\hline \multirow{2}{*}{\multicolumn{2}{|c|}{ Age (vears) }} & Group D $(n=55)$ & Group M $(n=55)$ & $95 \% \mathrm{CI}$ & p \\
\hline & & $46.62 \pm 8.272$ & $45.75 \pm 11.029$ & $-2.8,4.6$ & 0.640 \\
\hline \multirow{2}{*}{ Gender } & Male & $58.2 \%(32)$ & $52.7 \%(29)$ & & \multirow{2}{*}{0.565} \\
\hline & Female & $41.8 \%(23)$ & $47.3 \%(26)$ & & \\
\hline \multicolumn{2}{|c|}{ BMI $\left(\mathrm{kg} / \mathbf{m}^{2}\right)$} & $30.10 \pm 4.408$ & $29.77 \pm 4.378$ & $-1.3,2.0$ & 0.693 \\
\hline \multicolumn{2}{|c|}{ History of DM } & $3.6 \%(2)$ & $9.1 \%(5)$ & & 0.438 \\
\hline \multicolumn{2}{|c|}{ History of HTN } & $20.0 \%(11)$ & $18.2 \%(10)$ & & 0.808 \\
\hline \multicolumn{2}{|c|}{ Operative duration (minute) } & $160.91 \pm 29.644$ & $153.00 \pm 28.196$ & $-3.0,18.8$ & 0.155 \\
\hline
\end{tabular}

Although basal heart rate didn't show significant differences between both groups $(\mathrm{p}=0.290)$, most of the following readings showed significantly lower hear rates in group D in comparison with group $\mathrm{M}(\mathrm{p}<0.05)$ as illustrated in table (3).

Table (3): Basal and follow up values of HR (beat per minute) of the study groups

\begin{tabular}{|l|c|c|c|c|}
\hline \multicolumn{1}{|c|}{ Heart rate } & Group D $(\mathbf{n = 5 5})$ & Group $\mathbf{M}(\mathbf{n = 5 5})$ & $\mathbf{9 5 \%} \mathbf{C I}$ & $\mathbf{p}$ \\
\hline Basal & $85.38 \pm 7.529$ & $87.02 \pm 8.565$ & $-4.7,1.4$ & 0.290 \\
\hline Drug infusion & $82.04 \pm 7.338$ & $84.75 \pm 8.438$ & $-5.7,0.3$ & 0.075 \\
\hline Before intubation & $69.76 \pm 7.608$ & $82.62 \pm 8.489$ & $-15.9,-9.8$ & $<\mathbf{0 . 0 0 1}$ \\
\hline After intubation & $63.33 \pm 9.862$ & $83.75 \pm 8.765$ & $-23.9,-16.9$ & $<\mathbf{0 . 0 0 1}$ \\
\hline Speculum insertion & $63.07 \pm 9.903$ & $83.58 \pm 9.028$ & $-24.1,-16.9$ & $<\mathbf{0 . 0 0 1}$ \\
\hline $\mathbf{1 5}$ minutes & $62.96 \pm 10.132$ & $83.42 \pm 8.908$ & $-24.1,-16.8$ & $<\mathbf{0 . 0 0 1}$ \\
\hline $\mathbf{3 0}$ minutes & $63.20 \pm 10.853$ & $82.87 \pm 9.111$ & $-23.5,-15.9$ & $<\mathbf{0 . 0 0 1}$ \\
\hline $\mathbf{6 0}$ minutes & $62.91 \pm 10.814$ & $83.22 \pm 10.208$ & $-24.3,-16.3$ & $<\mathbf{0 . 0 0 1}$ \\
\hline $\mathbf{9 0}$ minutes & $62.67 \pm 10.844$ & $82.65 \pm 10.442$ & $-24.0,-16.0$ & $<\mathbf{0 . 0 0 1}$ \\
\hline $\mathbf{1 2 0}$ minutes & $62.61 \pm 10.931$ & $83.53 \pm 10.724$ & $-25.1,-16.7$ & $<\mathbf{0 . 0 0 1}$ \\
\hline $\mathbf{1 5 0}$ minutes & $62.83 \pm 11.878$ & $83.34 \pm 10.190$ & $-25.8,-15.3$ & $<\mathbf{0 . 0 0 1}$ \\
\hline $\mathbf{1 8 0}$ minutes & $62.80 \pm 14.088$ & $84.00 \pm 9.390$ & $-30.3,-12.1$ & $<\mathbf{0 . 0 0 1}$ \\
\hline $\mathbf{2 1 0}$ minutes & $55.00 \pm 24.042$ & $86.67 \pm 1.528$ & $-72.2,8.8$ & 0.089 \\
\hline Pre-extubation & $62.91 \pm 11.546$ & $82.76 \pm 10.770$ & $-24.1,-15.6$ & $<\mathbf{0 . 0 0 1}$ \\
\hline Post-extubation & $62.91 \pm 11.699$ & $84.80 \pm 11.697$ & $-26.3,-17.5$ & $<\mathbf{0 . 0 0 1}$ \\
\hline
\end{tabular}

MAP values didn't significantly differ between both groups either at the baseline or during drug infusion. However, the following readings showed that group D expressed significantly lower MAP values compared to the other group as shown in table (4). 
Table (4): Basal and follow-up values of MAP ( $\mathrm{mmHg}$ ) of the study groups

\begin{tabular}{|l|c|c|c|c|}
\hline \multicolumn{1}{|c|}{ MAP } & Group D $(\mathbf{n}=\mathbf{5 5})$ & Group $\mathbf{M}(\mathbf{n}=\mathbf{5 5})$ & $\mathbf{9 5 \%} \mathbf{C I}$ & $\mathbf{p}$ \\
\hline Basal & $98.18 \pm 4.750$ & $97.07 \pm 4.086$ & $-0.6,2.8$ & 0.192 \\
\hline Drug infusion & $94.27 \pm 5.523$ & $95.96 \pm 4.872$ & $-3.7,0.3$ & 0.091 \\
\hline Before intubation & $84.29 \pm 7.099$ & $88.93 \pm 5.878$ & $-7.1,-2.2$ & $<\mathbf{0 . 0 0 1}$ \\
\hline After intubation & $82.27 \pm 8.031$ & $91.71 \pm 7.305$ & $-12.3,-6.5$ & $<\mathbf{0 . 0 0 1}$ \\
\hline Speculum insertion & $77.85 \pm 7.663$ & $91.75 \pm 7.594$ & $-16.8,-11.0$ & $<\mathbf{0 . 0 0 1}$ \\
\hline $\mathbf{1 5}$ minutes & $77.78 \pm 7.932$ & $92.20 \pm 7.936$ & $-17.4,-11.4$ & $<\mathbf{0 . 0 0 1}$ \\
\hline $\mathbf{3 0}$ minutes & $77.91 \pm 8.307$ & $92.09 \pm 8.336$ & $-17.3,-11.0$ & $<\mathbf{0 . 0 0 1}$ \\
\hline 60 minutes & $77.96 \pm 8.583$ & $91.75 \pm 8.596$ & $-17.0,-10.5$ & $<\mathbf{0 . 0 0 1}$ \\
\hline $\mathbf{9 0}$ minutes & $77.85 \pm 8.864$ & $91.67 \pm 8.428$ & $-17.1,-10.5$ & $<\mathbf{0 . 0 0 1}$ \\
\hline $\mathbf{1 2 0}$ minutes & $77.86 \pm 8.591$ & $92.07 \pm 8.813$ & $-17.6,-10.9$ & $<\mathbf{0 . 0 0 1}$ \\
\hline $\mathbf{1 5 0}$ minutes & $77.93 \pm 8.658$ & $91.25 \pm 6.979$ & $-17.1,-9.6$ & $<\mathbf{0 . 0 0 1}$ \\
\hline $\mathbf{1 8 0}$ minutes & $79.40 \pm 7.500$ & $89.85 \pm 5.352$ & $-15.4,-5.5$ & $<\mathbf{0 . 0 0 1}$ \\
\hline $\mathbf{2 1 0}$ minutes & $80.00 \pm<0.001$ & $94.33 \pm 4.041$ & $-23.9,-4.7$ & $\mathbf{0 . 0 1 8}$ \\
\hline Pre-extubation & $77.82 \pm 9.145$ & $92.18 \pm 7.818$ & $-17.6,-11.1$ & $<\mathbf{0 . 0 0 1}$ \\
\hline Post-extubation & $81.93 \pm 10.274$ & $99.31 \pm 9.379$ & $-21.1,-13.7$ & $<\mathbf{0 . 0 0 1}$ \\
\hline
\end{tabular}

The mean values of Boezaart score were significantly lower in group D in comparison with group M (1.43 vs. 1.57 correspondingly-p=0.033). In addition, isoflurane and propranolol consumption showed a significant decrease in group D ( $\mathrm{p}<0.001$ ). Nevertheless, blood loss demonstrated no significant difference when comparing the same groups $(\mathrm{p}=0.093)$. Also, neither intraoperative fluid intake nor urine output was significantly different between both studied groups ( $\mathrm{p}=0.616$ and 0.225 correspondingly). Additionally, group $\mathrm{M}$ expressed significantly shorter emergence and extubation times compared to group D. However, cases in the same group expressed significantly higher VAS values $(\mathrm{p}=0.018))$ as illustrated in table (5).

Table (5): Intra-operative isoflurane, propranolol and nitroglycerine consumption, Boezaart score, fluid intake, urine output, blood loss, emergence and extubation times, post-operative VAS score and morphine requirements of the groups

\begin{tabular}{|l|c|c|c|c|}
\hline & Group D $(\mathbf{n}=\mathbf{5 5})$ & Group $(\mathbf{n}=\mathbf{5 5})$ & $\mathbf{9 5 \%} \mathbf{C I}$ & $\mathbf{p}$ \\
\hline Boezaart & $1.43 \pm 0.4325$ & $1.57 \pm 0.369$ & $-0.3,0.0$ & $\mathbf{0 . 0 3 3}$ \\
\hline Isoflurane consumption (ml) & $51.64 \pm 11.509$ & $61.45 \pm 15.446$ & $-14.97,-4.67$ & $<\mathbf{0 . 0 0 1}$ \\
\hline $\begin{array}{l}\text { Propranolol consumption/per } \\
\text { patient }\end{array}$ & $0.31 \pm 0.245$ & $0.96 \pm 0.383$ & $-0.78,-0.53$ & $<\mathbf{0 . 0 0 1}$ \\
\hline Intraoperative fluid intake (mI) & $1556.36 \pm 376.02$ & $1522.73 \pm 322.72$ & $-98.8,166.1$ & 0.616 \\
\hline Urine output (ml) & $740.91 \pm 230.55$ & $692.73 \pm 180.12$ & $-30.0,126.4$ & 0.225 \\
\hline Blood loss (ml) & $230.45 \pm 52.19$ & $249.09 \pm 62.54$ & $-40.4,3.1$ & 0.093 \\
\hline Emergence time (minute) & $10.27 \pm 2.670$ & $7.47 \pm 2.176$ & $1.9,3.7$ & $<\mathbf{0 . 0 0 1}$ \\
\hline Extubation time (minute) & $13.62 \pm 2.812$ & $10.58 \pm 2.580$ & $2.0,4.1$ & $<\mathbf{0 . 0 0 1}$ \\
\hline VAS at discharge from PACU & 2.18 & 1.85 & $0.1,0.6$ & 0.018 \\
\hline Time to discharge & $38.3(5.2)$ & $60.7(6.8)^{*}$ & $0.02,0.82$ & $\mathbf{0 . 0 4 0}$ \\
\hline
\end{tabular}

\section{DISCUSSION}

As earlier clinical investigations have pointed to the efficacy of dexmedetomidine as a sedative in cases with critical illness, newer researches have focused on the efficacy of alpha-2 receptor agonists as adjuvants to neuroanesthesia. Dexmedetomidine has multiple favorable clinical effects including hemodynamic stability, neuroprotection, and lack of respiratory depression without any interference with intraoperative neurophysiological monitoring. These suggested that dexmedetomidine could be useful in terms of neurosurgical patients treatment ${ }^{(14)}$.

The current study was carried out at to compare between dexmedetomidine and $\mathrm{MgSO}_{4}$ on the adequacy of hypotensive anesthesia during transsphenoidal excision of pituitary tumors. A total of 110 cases was enrolled, and they were divided into 2 groups: Group D
(55 cases) that was commenced on dexmedetomidine, and group F (55 cases), which received fentanyl.

No significant changes were detected between both studied groups as regards demographic characteristics, and this should nullify any bias that may have skewed results in favor of one group rather than the other one. Another study that evaluated the efficiency of dexmedetomidine in maintaining hemodynamic stability in cases with pituitary surgery also reported no significant change among both studied groups as regards patient' demographics $(\mathrm{p}>0.05)^{(9)}$.

The present study demonstrated that group D tended to have lower heart rates and mean arterial pressures compared to group $\mathrm{M}$ although no significant difference existed regarding their baseline values. Similarly, Bala et al. ${ }^{(\mathbf{9})}$ recorded that dexmedetomidine administration was associated with a significant 
decrease in both MAP and HR compared to controls in spite of being comparable at baseline. Batra et al. ${ }^{(1)}$ also reported that both heart rate and BP readings had lower values in the dexmedetomidine group in comparison with controls throughout most intraoperative readings although both groups had no significant difference prior to operation. Moreover, other researches have confirmed our findings as regards the effectiveness of dexmedetomidine in maintaining hemodynamic stability in pituitary surgeries ${ }^{(15,16)}$.

Cardiovascular response in the form of tachycardia and hypertension is occasionally encountered in multiple intracranial surgeries. About 50 - $90 \%$ of such cases will require perioperative antihypertensive agents to control blood pressure ${ }^{(17,18)}$. Due to severe nociceptive stimuli experienced by the patients during trans-sphenoidal pituitary surgeries, the anesthesiologist often encounters perturbations in heart rate and BP during multiple surgical stages. This will require increasing the anesthesia or increasing opioid administration, which was demonstrated to be associated with hypotension with compromise of the cerebral circulation. Besides, these maneuvers are associated with prolonged recovery time ${ }^{(15,19)}$. A previous Egyptian study has compared dexmedetomidine to fentanyl in cochlear implant surgery. Authors reported that group D expressed significantly lower heart rates and MAP during operation compared to the fentanyl group ${ }^{(20)}$. This also coincides with our results.

Multiple previous researches have confirmed the efficacy of dexmedetomidine in attenuating the changes of intraoperative hemodynamics. Therefore, it gained a great popularity in neurosurgical procedures ${ }^{(21-23)}$. These effects could be clarified by its central and peripheral actions. With regard to CNS, it decreases the sympathetic outflow. Additionally, it blocks peripheral ganglia. Both of the previous mechanisms could explain its protective effect on hemodynamic changes during neurosurgical procedures ${ }^{(9)}$. The previous reports emphasize the importance of dexmedetomidine as adjunctive to general anesthesia in such cases, as it decreases the occurrence of hypertensive episodes, which can lead to bleeding, edema, worsening of surgical field, and increase of intracranial pressure ${ }^{(18)}$.

In the current study, the mean values of Boezaart score were significantly decreased in group D in comparison with group $\mathrm{M}(1.43$ vs. 1.57 correspondingly $-p=0.033$ ). Likewise, El Saied and his colleagues ${ }^{(20)}$ reported that the surgical field quality was significantly better in the dexmedetomidine group $(\mathrm{p}=0.011)$. Quality scale had mean values of 2.19 and 2.76 in the dexmedetomidine and fentanyl groups correspondingly. Other authors reported that dexmedetomidine administration was associated with significantly higher surgeon satisfaction ${ }^{(16)}$. These effects are secondary to better hypotensive anesthesia with dexmedetomidine, which leads to decrease tissue oozing during surgical dissection especially in narrow surgical fields like that encountered in neurosurgical practice. In accordance Faranak and his colleagues ${ }^{(24)}$ have demonstrated that blood loss was minimal and the surgeon's satisfaction score was greater in the dexmedetomidine group in comparison with $\mathrm{MgSO}_{4}$ group (24). Also, Bayram and his colleagues (25) compared the efficiency of $\mathrm{MgSO}_{4}$ and dexmedetomidine in the context of hypotension in FESS operations and demonstrated that dexmedetomidine was associated with a greater degree of surgeon's satisfaction in comparison with the $\mathrm{MgSO}_{4}$ group $^{(25)}$

In our study, group D had significantly lower isoflurane consumption in comparison with group $\mathrm{M}$ (51.64 vs. $61.45 \mathrm{ml}$ correspondingly $-\mathrm{p}<0.001$ ). Dexmedetomidine is associated with decreased inhalation anesthetic requirement because of its alphaII agonist actions that suppress norepinephrine transmission ${ }^{(26,27)}$. Its sedative effect is associated with a $35-50 \%$ reduction in intraoperative isoflurane requirements ${ }^{(28)}$. This was documented in three previous researches that reported that Dexmedetomidine administration led to decreased inhalation anesthetic intake ${ }^{(9,29,30)}$. Of course, this will lead to a faster and better recovery from anesthesia, which will allow early assessment of the patient's neurological functions after operation.

Although statistical analysis didn't reveal any significant change among both studied groups in the context of intraoperative blood loss $(\mathrm{p}=0.93)$, group $\mathrm{D}$ tended to have less blood loss during surgery (230.45 vs. $249.09 \mathrm{ml}$ in group M). Bala and his associates ${ }^{(9)}$ also negate any significant difference among both groups as regards estimated blood loss. However, it had mean values of 153.3 and $218 \mathrm{ml}$ in the dexmedetomidine and control groups respectively. Despite its statistical insignificance, it was evident that blood loss was higher in the controls ${ }^{(9)}$. Conversely, another study reported that dexmedetomidine was accompanied by a significant decrease in intraoperative bleeding $(p=0.012)$. It had mean values of 160 and 305 $\mathrm{ml}$ in dexmedetomidine and control groups correspondingly ${ }^{(16)}$.

We have demonstrated that, propranolol administration was significantly decreased in group D compared to group $\mathrm{M}$ ( 0.31 vs. 0.96 correspondingly $\mathrm{p}<0.001)$. In another randomized study, dexmedetomidine infusion was accompanied by a significant decrease in the administration of antihypertensive medications comprising beta blockers and hydralazine ${ }^{(23)}$. This comes in line with our findings.

Our findings revealed that group D expressed significantly longer emergence and extubation times compared to group $M$. Both early emergence and tracheal extubation are crucial for neurosurgical anesthesia. These parameters were significantly increased with dexmedetomidine. In agreement, Faranak and his colleagues ${ }^{(24)}$ have demonstrated that 
cases in the dexmedetomidine group were more sedated at the PACU. In addition, the period to reach MAS $\geq$ nine was longer in comparison to those of the $\mathrm{MgSO}_{4}$ group. Also, Erdem and his colleagues ${ }^{(31)}$ have displayed that, the sedation score was greater when dexmedetomidine was given to induce hypotension throughout FESS in comparison to esmolol. Gunes and his associates (32) noted delayed recovery after intracranial surgery in patients administered dexmedetomidine. Tanskanen and his colleagues (18) were on contrary and noted that the dexmedetomidine infusion was associated with faster recovery from GA. They also reported no cases with respiratory depression that may delay patient recovery. It should be noted that this difference between researches could be attributed to the different dose and infusion rate. Higher doses might have caused over sedation, which led to delayed recovery.

In the present study, the postoperative VAS pain score in $\mathrm{D}$ group was significantly less than in $\mathrm{M}$ group. Thus, dexmedetomidine was demonstrated to have a better analgesic effect in companion with $\mathrm{MgSO}_{4}$. In concurrence to these results Faranak et al. (24) study showed less analgesic was needed in the dexmedetomidine group in comparison with the $\mathrm{MgSO}_{4}$ group.

Dong and his colleagues ${ }^{(33)}$ evaluated the effect of adding dexmedetomidine to a sufentanil-based analgesics for postsurgical pain management in the context of spine surgeries, and they demonstrated that dexmedetomidine was associated with a minimal opioid requirement and satisfactory pain management throughout postsurgical period. On the other hand, Abo shanab and his colleagues ${ }^{(34)}$ conducted a study to compare between both medications in the context of middle ear surgery. They reported that only $16 \%$ of patients in the $\mathrm{MgSO}_{4}$ group and $14 \%$ of cases in the dexmedetomidine group needed rescue analgesics. Such outcomes could be clarified by the analgesic effects of both studies' medications ${ }^{(34)}$. Peng and his colleagues ${ }^{(35)}$ evaluated the effect of $\mathrm{IV} \mathrm{MgSO}_{4}$ on postsurgical analgesia for orthopedic surgeries and reported that perioperative IV administration of $\mathrm{MgSO}_{4}$ could decrease postsurgical analgesic consumption and decrease postsurgical pain ${ }^{(35)}$. The analgesic effects of magnesium are owing to blocking $\mathrm{Ca}^{++}$channels and antagonism of the NMDA receptor and enhancement of opioids effect in the CNS. This effect was first noticed in patients with malignant tumours managed with morphine. These different results could be explained by the difference between researches in the dose and infusion rate of the studied drugs. Even both drugs had analgesic effect. It appears that dexmedetomidine has a more potent analgesic effect in comparison with $\mathrm{MgSO}_{4}$.

Our study had multiple limitations where it had a small sample size. Also, the efficacy of dexmedetomidine in cases with pre-existing heart disease should be researched. In addition, stress hormone levels (like cortisol) should be evaluated as well. These drawbacks should be well covered in the upcoming researches.

\section{CONCLUSION}

Based on our findings, Dexmedetomidine appears to be superior compared to magnesium sulphate in achieving hypotensive anesthesia during pituitary surgery. It is associated with lower heart rate, mean arterial pressure, Boezaart score. It also had better postoperative analgesic effect while magnesium sulphate had better recovery.

\section{Conflict of Interest: Nil. \\ Funding: Nil.}

\section{REFERENCES}

1. Batra A, Verma R, Bhatia V et al. (2017): Dexmedetomidine as an anesthetic adjuvant in intracranial surgery. Anesthesia, essays and researches, 11 (2): 309.

2. Horvat A, Kolak J, Gopčević A et al. (2011): Anesthetic management of patients undergoing pituitary surgery. Acta Clinica Croatica, 50 (2): 209-16.

3. Nemergut E, Dumont A, Barry U et al. (2005): Perioperative management of patients undergoing transsphenoidal pituitary surgery. Anesthesia \& Analgesia, 101 (4): 1170-81.

4. Lim M, Williams D, Maartens N (2006): Anaesthesia for pituitary surgery. Journal of clinical neuroscience, 13(4): 413-8.

5. Shams T, El Bahnasawe N, Abu-Samra M et al. (2013): Induced hypotension for functional endoscopic sinus surgery: A comparative study of dexmedetomidine versus esmolol. Saudi journal of anaesthesia, 7 (2): 175.

6. Lee S (2019): Dexmedetomidine: present and future directions. Korean Journal of Anesthesiology, 72 (4): 323.

7. Aresta M, Dibenedetto A, Quaranta E (2016): State of the art and perspectives in catalytic processes for $\mathrm{CO} 2$ conversion into chemicals and fuels: The distinctive contribution of chemical catalysis and biotechnology. Journal of Catalysis, 343: 2-45.

8. Srivastava V, Mishra A, Agrawal S et al. (2016): Comparative evaluation of dexmedetomidine and magnesium sulphate on propofol consumption, haemodynamics and postoperative recovery in spine surgery: a prospective, randomized, placebo controlled, double-blind study. Advanced pharmaceutical bulletin, 6 (1): 75.

9. Bala R, Chaturvedi A, Pandia M et al. (2019): Intraoperative dexmedetomidine maintains hemodynamic stability and hastens postoperative recovery in patients undergoing transsphenoidal pituitary surgery. Journal of neurosciences in rural practice, 10 (4): 599.

10.Boezaart A, van der Merwe J, Coetzee A (1995): Comparison of sodium nitroprusside-and esmololinduced controlled hypotension for functional endoscopic sinus surgery. Canadian journal of anaesthesia, 42 (5): 373-6.

11.Biro P (2014): Calculation of volatile anaesthetics consumption from agent concentration and fresh gas flow. Acta anaesthesiologica Scandinavica, 58 (8): 968-72.

12. Heller G, Manuguerra M, Chow R (2016): How to analyze the visual analogue scale: myths, truths and 
clinical relevance. Scandinavian Journal of Pain, 13: 6775.

13.Aldrete J (1995): The post-operative recovery score revisited. J Clin Anesth., 7 (1): 89-91.

14.Schwarz A, Nossaman B, Carollo D et al. (2013): Dexmedetomidine for neurosurgical procedures. Current Anesthesiology Reports, 3 (3): 205-9.

15. Gopalakrishna K, Dash P, Chatterjee N et al. (2015): Dexmedetomidine as an anesthetic adjuvant in patients undergoing transsphenoidal resection of pituitary tumor. Journal of neurosurgical anesthesiology, 27 (3): 209-15.

16.Salimi A, Sharifi G, Bahrani H et al. (2017): Dexmedetomidine could enhance surgical satisfaction in trans-sphenoidal resection of pituitary adenoma. J Neurosurg Sci., 61 (1): 46-52.

17.Sturaitis M, Kroin J, Swamidoss C et al. (2002): Effects of intraoperative dexmedetomidine infusion on hemodynamic stability during brain tumor resection. Anesthesiology, 97 (3A): A310.

18. Tanskanen $P$, Kyttä J, Randell $T$ et al. (2006): Dexmedetomidine as an anaesthetic adjuvant in patients undergoing intracranial tumour surgery: a double-blind, randomized and placebo-controlled study. BJA: British Journal of Anaesthesia, 97 (5): 658-65.

19.Ali Z, Prabhakar H, Bithal P et al. (2009): Bispectral index-guided administration of anesthesia for transsphenoidal resection of pituitary tumors: a comparison of 3 anesthetic techniques. Journal of neurosurgical anesthesiology, 21 (1): 10-5.

20.El Saied M, Mohamed N, Mohamed H et al. (2016): Dexmedetomidine versus fentanyl in anesthesia of cochlear implantation in pediatric patients. Egyptian Journal of Anaesthesia, 32 (1): 55-9.

21. Yildiz M, Tavlan A, Tuncer S et al. (2006): Effect of dexmedetomidine on haemodynamic responses to laryngoscopy and intubation. Drugs in R \& D., 7 (1): 4352.

22. Uyar A, Yagmurdur H, Fidan Y et al. (2008): Dexmedetomidine attenuates the hemodynamic and neuroendocrinal responses to skull-pin head-holder application during craniotomy. Journal of neurosurgical anesthesiology, 20 (3): 174-9.

23. Bekker A, Sturaitis M, Bloom M et al. (2008): The effect of dexmedetomidine on perioperative hemodynamics in patients undergoing craniotomy. Anesthesia \& Analgesia, 107 (4): 1340-7.

24. Rokhtabnak F, Motlagh S, Ghodraty M et al. (2017): Controlled hypotension during rhinoplasty: A comparison of dexmedetomidine with magnesium sulfate. https://pubmed.ncbi.nlm.nih.gov/29696129
25. Bayram A, Ülgey A, Günes I et al. (2015): Comparison between magnesium sulfate and dexmedetomidine in controlled hypotension during functional endoscopic sinus surgery. Revista brasileira de anestesiologia, 65: 617.

26. Muniyappa R, Rajappa G, Govindswamy S et al. (2016): Effect of dexmedetomidine bolus dose on isoflurane consumption in surgical patients under general anesthesia. Anesthesia, essays and researches, 10 (3): 649.

27. Weerink M, Struys M, Hannivoort L et al. (2017): Clinical pharmacokinetics and pharmacodynamics of dexmedetomidine. Clinical Pharmacokinetics, 56 (8): 893-913.

28. Gertler R, Brown H, Mitchell D et al. (2001): Dexmedetomidine: a novel sedative-analgesic agent. Baylor University Medical Center Proceedings, Taylor \& Francis.

29. Nunes R, Cavalcante S (2002): Influence of dexmedetomidine upon sevoflurane end-expiratory concentration: evaluation by bispectral index, suppression rate and electroencephalographic power spectral analysis. Revista brasileira de anestesiologia, 52 (2): 133-45.

30. Magalhães E, Govêia CS, Ladeira L et al. (2004): Relationship between dexmedetomidine continuous infusion and end-tidal sevoflurane concentration, monitored by bispectral analysis. Revista brasileira de anestesiologia, 54 (3): 303-10.

31. Erdem A, Kayabasoglu G, Tuna A et al. (2016): Effect of controlled hypotension on regional cerebral oxygen saturation during rhinoplasty: a prospective study. Journal of clinical monitoring and computing, 30 (5): 655-60.

32. Günes Y, Gündüz M, Özcengiz D et al. (2005): Dexmedetomidine-remifentanil or propofol-remifentanil anesthesia in patients undergoing intracranial surgery. Neurosurgery Quarterly, 15 (2): 122-6.

33. Dong C, Lu Y, Zhang J et al. (2016): The optimal dose of dexmedetomidine added to an sufentanil-based analgesic regimen for postoperative pain control in spine surgery: a probit analysis study. europepmc.org/articles/PMC5265895

34. Aboushanab O, El-Shaarawy A, Omar A et al. (2011): A comparative study between magnesium sulphate and dexmedetomidine for deliberate hypotension during middle ear surgery. Egyptian journal of anaesthesia, 27 (4): 227-32.

35.Peng Y, Sung F, Huang M et al. (2018): The use of intravenous magnesium sulfate on postoperative analgesia in orthopedic surgery: A systematic review of randomized controlled trials. Medicine, 97 (50): e13583. 